\title{
Progressive neuronal degeneration of childhood with liver disease (Alpers' disease) presenting in young adults
}

\author{
B N Harding, N Alsanjari, S J M Smith, C M Wiles, D Thrush, D H Miller, F Scaravilli, \\ A E Harding
}

\begin{abstract}
Two unrelated and previously healthy girls, aged 17 and 18, presented with a subacute encephalopathy, visual and sensory symptoms and signs, and prominent seizures that were difficult to control. Brain MRI showed lesions (high signal on $T 2$ weighted images) in the occipital lobes and thalamus; EEG showed slow wave activity with superimposed polyspikes. Inexorable downhill progression led to death in hepatic failure within eight months of onset. Histopathological findings in both patients $((a)$ chronic hepatitis with prominent bile duct proliferation, fatty change, and fibrosis; (b) in the brain a patchy destruction of the cerebral cortex, predominantly involving striate cortex) were characteristic of progressive neuronal degeneration of childhood with liver disease-Alpers-Huttenlocher syndrome-a rare autosomal recessive disorder usually seen in infants and young children.
\end{abstract}

(F Neurol Neurosurg Psychiatry 1995;58:320-325)

National Hospital for Neurology and Neurosurgery, Queen Square, London, UK Department of Neuropathology B N Harding

N Alsanjari

F Scaravilli

Department of Clinical

Neurophysiology

S J M Smith

Department of

Clinical Neurology

C M Wiles

D H Miller

A E Harding

Derriford Hospital,

Plymouth, UK

D Thrush

Correspondence to:

Dr B N Harding,

Department of

Histopathology

Neuropathology), Great

(Neuropathology), Great

Ormond Street Hospital for

Children NHS Trust, Great

WCIN 3JH, UK.

Received 17 May 1994

and in revised form

20 October 1994.

Accepted 1 November 1994
Keywords: progressive neuronal degeneration of childhood; liver disease; Alpers' disease

Progressive neuronal degeneration of childhood with liver disease (PNDC), a rare autosomal recessive neurodegenerative disorder of uncertain aetiology, usually causes a characteristic clinical triad of psychomotor retardation, intractable epilepsy, and liver failure in infants and young children. ${ }^{1-3}$ Definitive diagnosis requires postmortem examination of the brain and liver, but recent correlative clinicopathological studies at the Hospital for Sick Children, London, indicate electrophysiological, radiological, and biochemical abnormalities sufficiently characteristic to suggest the diagnosis during life..$^{4-7}$ PNDC is usually rapidly fatal. Few patients survive into their teens, so it is not normally considered in the differential diagnosis of epilepsy with first presentation outside childhood. Our recent experience with two cases of PNDC, similar to the childhood disorder, but occurring in unrelated and previously healthy young women who presented initially with seizures at 17 and 18 years of age, has prompted a reappraisal of this view and is the subject of this report.

\section{Case reports}

CASE 1

This young woman, who was entirely normal until 18 years of age, suddenly saw flashing lights and had impaired vision in both upper visual fields. The teichopsia resolved after three days but blurred vision persisted and she complained of headache. Next day she had a generalised seizure: examination showed pendular nystagmus and left homonymous hemianopia, but no other abnormal findings. Routine haematological and biochemical investigations including liver function tests, brain CT, carotid angiography and CSF examination were normal. An EEG showed almost continuous polymorphic slow waves $(2 / s)$ and polyspikes over the posterior part of the hemisphere (fig 1). She was treated with carbamazepine; her alanine aminotransferase was marginally increased on this, but bilirubin and alkaline phosphatase were normal. She then started to have frequent focal seizures, with clonic jerking of the left arm and deviation of the eyes to the left. Positive visual symptoms recurred. The EEG had deteriorated with polyspike and slow wave activity now over the left hemisphere, although the right sided predominance of the abnormality persisted. Repeat CT showed an ill defined low density lesion in the right occipital lobe, mainly involving white matter. Brain MRI showed increased signal in the medial temporal and occipital lobes on the right on $\mathrm{T} 2$ weighted images. She then developed multifocal myoclonus and a left lateral gaze palsy. Three weeks after admission she had five generalised seizures, and one week later continuous left sided jerking with confusion and drowsiness. There was cervical lymphadenopathy. She developed a left hemiparesis; plantar responses were extensor. A further CT was normal, but MRI showed bilateral areas of high signal in the occipital lobes and thalamus (fig 2). Pattern visual evoked potentials were of normal latency, but asymmetric, with smaller responses over the right hemisphere. Sensory nerve action potentials were reduced in amplitude. She was treated with intravenous acyclovir. Routine blood investigations and repeat CSF examination remained normal; blood and CSF viral titres were normal. Her seizures did not respond to intravenous phenytoin or chlormethiazole, but were partly suppressed by phenobarbitone and carbamazepine.

She made a partial recovery, with resolution of her field defect to a left lower 
Figure 1 EEG five days after presentation in case 1. High amplitude polymorphic slow activity and polyspikes are seen over the posterior part of the right hemisphere. Activity over the left hemisphere is relatively well preserved.

FP2-F8

F8-T4

T4-T6

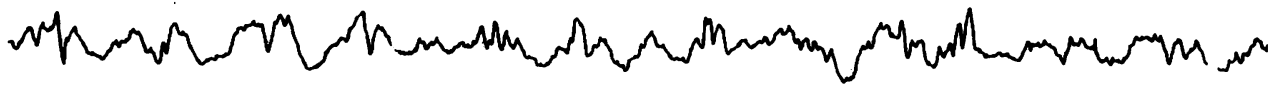
T6-O2

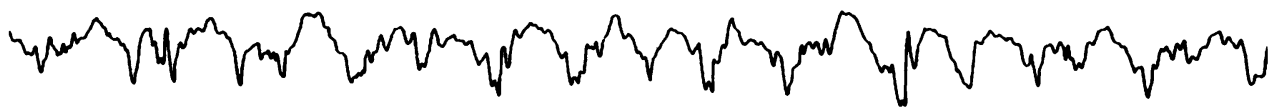

FP1-F7 F7-T3 T3-T5

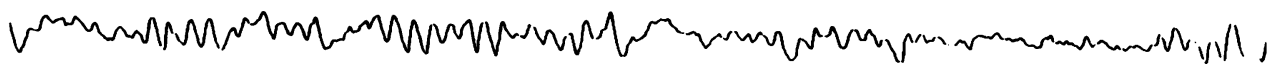

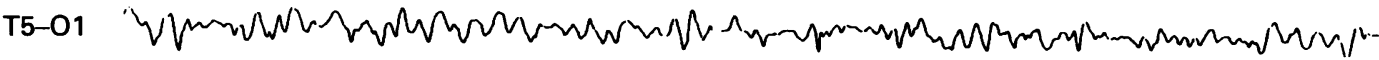
F8-F4

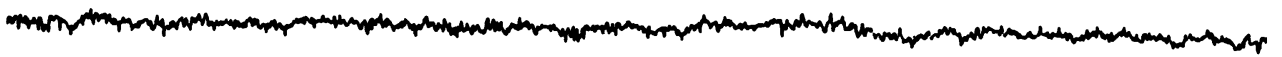
F4-Fz Fz-F3 F3-F7 T6-P4

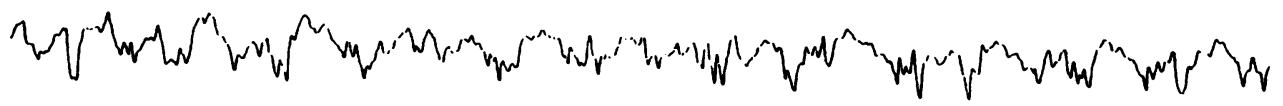

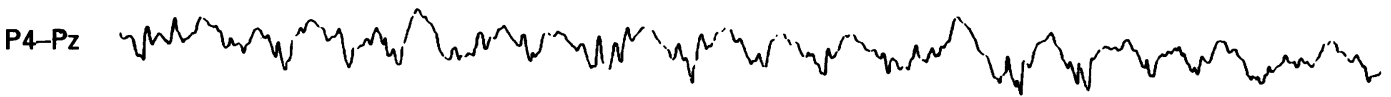

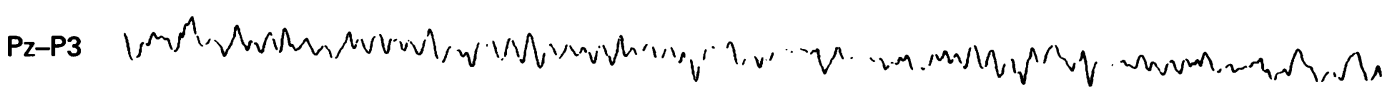
P3-T5 MF $70 \mathrm{~Hz}$

quadrantanopia, and less frequent jerks, occurring in the left leg only. Partial and generalised seizures recurred and she was treated with sodium valproate ( $1.5 \mathrm{~g}$ daily). Again she improved but then became aware of further visual loss, with acuities of counting fingers only; this appeared to be cortical in origin. Four months after the onset of her symptoms she again developed recurrent generalised and focal (left sided) seizures despite treatment with phenytoin, clobazam, phenobarbitone, and sodium valproate. An EEG showed continuous polyspike and slow wave activity, maximal over the right temporo-occipital area. Brain MRI showed similar but more extensive high signal lesions. Her worsening seizures were treated initially with intravenous chlormethiazole, which had little effect. Treatment with sodium valproate, clobazam, and phenytoin were stopped, she was electively intubated, ventilated, and treated with intravenous thiopentone as well as her baseline phenobarbitone. This was continued, monitoring thiopentone and pentobarbitone concentrations for five days with a resulting burst suppression pattern of isoelectric EEG. Two days after cessation of thiopentone infusion she became oedematous, and developed adult respiratory distress syndrome, followed in quick succession by a clotting disorder, an acute abdomen, with clinical and biochemical features of acute pancreatitis, and a tendency to have hypoglycaemic episodes. Within three days she became jaundiced and clearly had acute hepatic failure, which became complicated by impaired renal function. Intensive medical treatment was to no avail and she 
Figure $2 T 2$ weighted brain MRI image in case 1 ; axial image showing bilateral thalamic and right occipital cortex lesions.

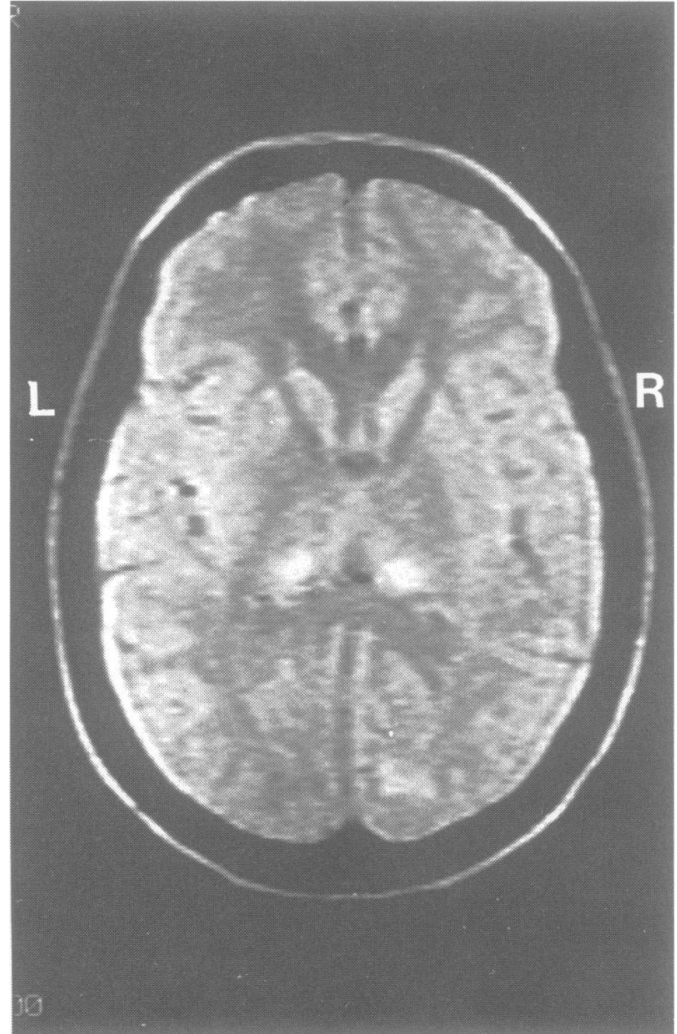

died six months after the onset of her symptoms.

\section{Pathological features}

Her fixed brain weighed $1250 \mathrm{~g}$. Examination of the coronal slices showed that a haemorrhage $2 \times 2 \times 2 \mathrm{~cm}$ had destroyed the left thalamus and compressed the left lateral ventricle. On the right side the parietal cortex showed laminar softening and the whole calcarine cortex was thin, granular, and brownish grey (fig 3), with similar patches of abnormal cortex extending into medial superior and lateral occipital areas. Additionally, within the white matter core of the central part of the right occipital lobe towards the pole there was a sharply defined zone of tissue breakdown, $1.5 \times 2.5 \times 2 \mathrm{~cm}$. The cortex and white matter in the left hemisphere appeared normal.

Microscopically, the cerebral cortex from the left hemisphere and most of the right were unaffected except for Alzheimer type II astrocytosis superficially. The right striate cortex (fig 4) was thinned but loose in texture except superficially where it was intensely gliotic; the residuum was devoid of neurons, and composed of hypertrophic fibre forming astrocytes. Similar patches of devastated cortex were present in other parts of the right occipital and parietal lobes, as well as other foci showing less severe involvement, either superficial astrocytosis and fine sponginess in layer II, or more notable neuronal loss extending into layers III and IV. The macroscopically abnormal right occipital white matter comprised myelin fragments, foamy macrophages, reactive fibre forming astrocytes, and capillary vessels. Beneath the affected parietal cortex the subcortical white matter was partly demyelinated and gliotic. The left thalamus contained acute haemorrhage with surrounding oedema. Most of the right thalamus showed pronounced spongiosis (fig 5A), considerable neuronal loss including some mineralised cell remnants, and astrocytosis. Both lateral geniculate nuclei showed moderate diffuse neuronal loss. Bilaterally the cerebellar dentate nuclei were spongy, gliotic, and depleted of nerve cells. Large areas of subcortical and foliate white matter exhibited a fine spongiosis and gliosis associated with some Purkinje cell and granule cell loss from the supra-adjacent cortex (fig 5B); these abnormalities were present on both superior and inferior aspects of the hemispheres away from the vascular boundary zones. Hepatic findings (fig 6) were fibrosis, nodular regeneration, pronounced bile duct proliferation, fatty infiltration, and bile stasis. Similar abnormalities, but lacking bile duct proliferation, were noted on liver biopsy two months before death.

CASE 2

This young woman, aged 17 , was well until seven months before admission to the National Hospital when she had had a two hour episode of altered sensation on the right side, with weakness of the right leg. Her motor and intellectual development had been entirely normal. She occasionally used recreational drugs. One month later she presented with jerking of the right leg that lasted for several hours, followed by a generalised tonic clonic seizure. Brain CT and EEG were normal, and she was treated with carbamazepine. She had no further symptoms for four months but then had blurred vision on the left side, nausea, headache, focal seizures involving the left arm, and three generalised seizures. On examination she had a left lower quadrantanopia and impaired joint position sense in the left toes. Over the next month she had virtually continuous twitching of the left sternomastoid and arm, occasionally affecting the
Figure 3 Case 1: coronal slices at the level of the occipital lobes. On the right side the calcarine and adjacent medial occipital cortex is thinned, granular, and discoloured. There is also tissue breakdown within the central white matter. 


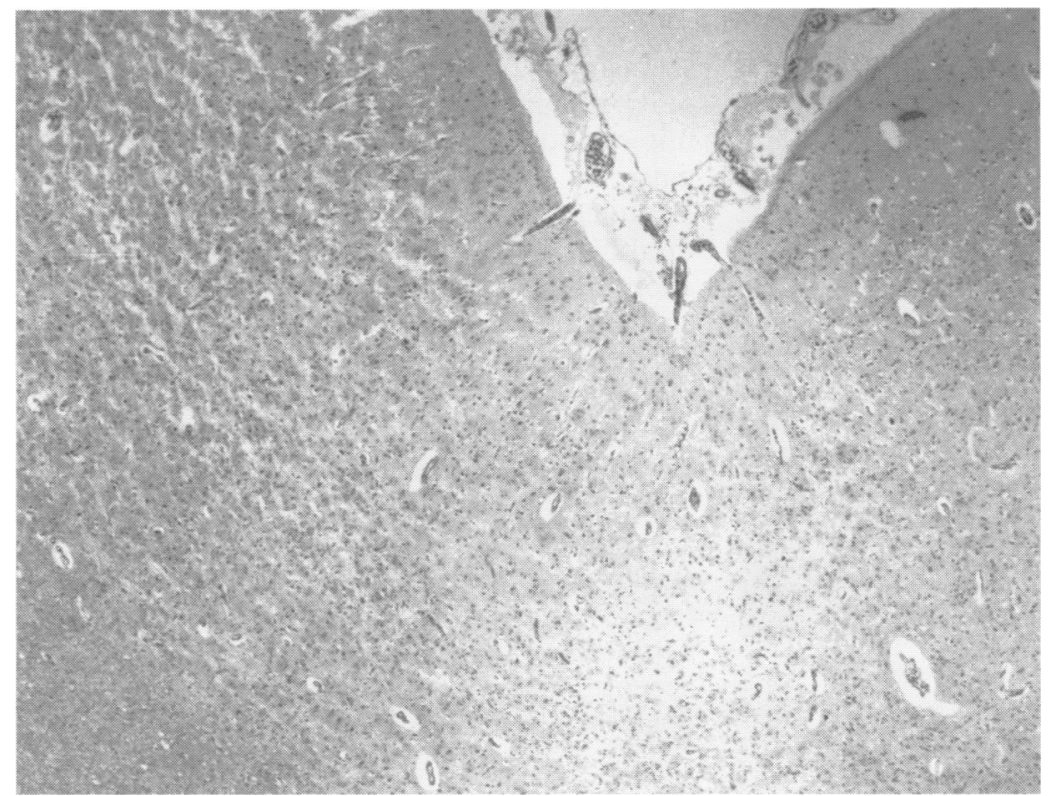

Figure 4 Case 1: microscopic section of the right striate cortex showing collapse and destruction of the neuropil and replacement by hypertrophic astrocytes. Haematoxylin-eosin originally $\times 45$.
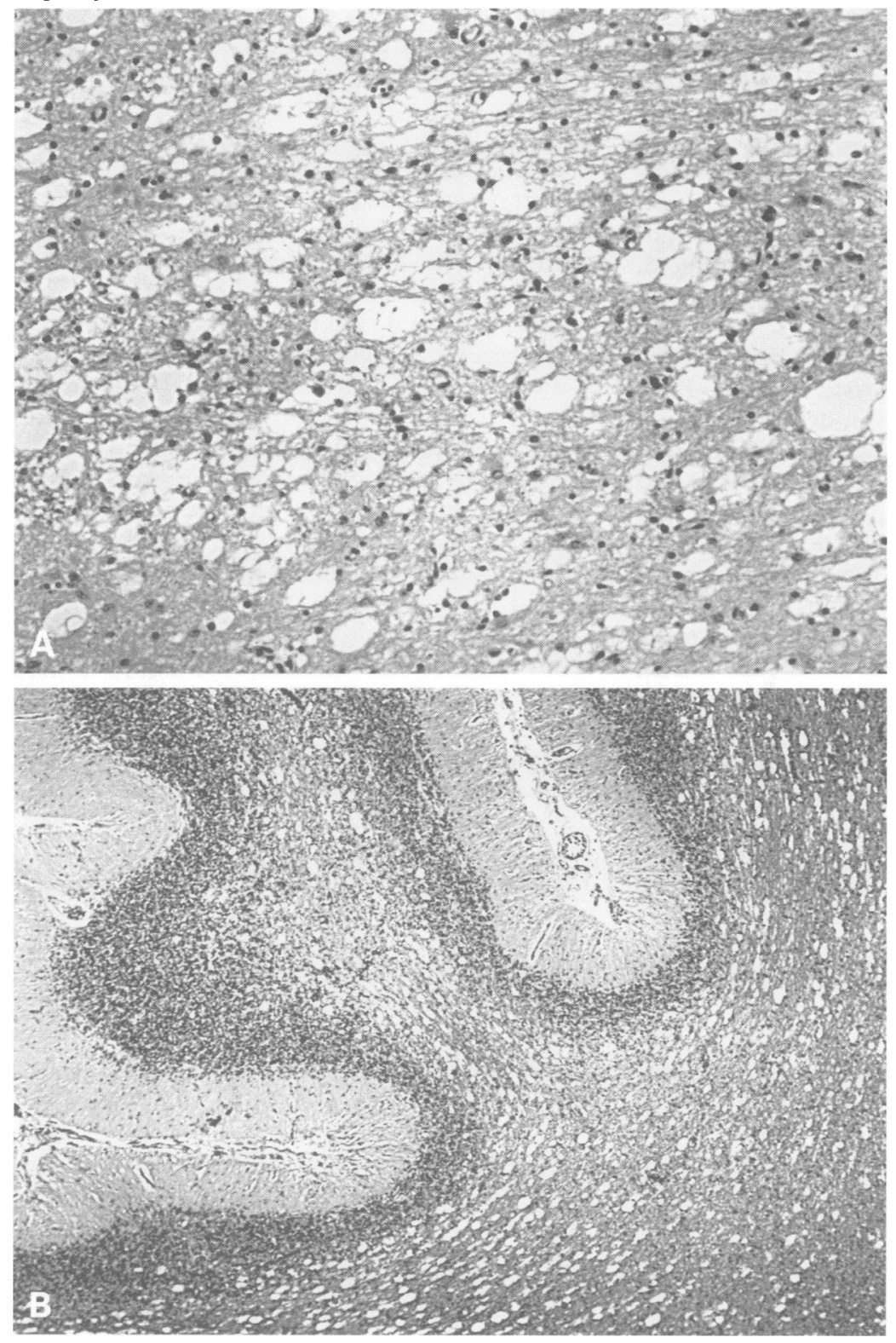

Figure 5(A) Case 1: spongiosis and neuronal loss are prominent features in the right thalamus. Haematoxylin-eosin originally $\times 180$. (B) Case 1: spongiosis is also extensive in cerebellar subcortical and foliate white matter along with moderate Purkinje cell depletion. Haematoxylin-eosin originally $\times 45$. left leg, and developed left homonymous hemianopia and hemianaesthesia with mild hemiparesis. She had intermittent visual hallucinations. Further CT showed a low density area in the right occipital region; MRI showed increased signal in the right occipital pole and thalamus. An EEG showed polyspikes in the right parieto-occipital region and bilateral excess slow activity. Routine haematological and biochemical investigations were normal, including liver function tests. Her CSF was acellular but the protein was increased on two occasions $(1.56$ and $1.82 \mathrm{~g} / \mathrm{l})$. Viral titres in CSF and serum were unremarkable. Plasma lactate concentration was normal. Clonazepam, lamotrigine, and vigabatrin failed to control her focal seizures.

Seven months after the onset of this illness, she developed status epilepticus requiring intravenous barbiturate treatment and ventilation. One week later she was drowsy but responded to commands. There were divergent strabismus with left conjugate gaze palsy, also intermittent jerks of all limbs and asymmetric tetraparesis, both more pronounced on the left. Reflexes were absent and plantar responses flexor. General examination was normal. She was treated with sodium valproate $(3 \mathrm{~g}$ ) and clonazepam ( $2 \mathrm{mg}$ daily). Extensive investigations included blood ammonias (normal throughout admission except once at $156 \mathrm{mmol} / \mathrm{l}$ ), slightly increased blood but normal CSF lactate concentrations, negative blood and CSF viral titres, and normal white cell enzymes, urinary and amino and organic acids, and creatine kinase. Muscle biopsy showed pronounced type 2B fibre atrophy but no ragged red or cytochrome oxidase negative fibres, and mitochondria were ultrastructurally normal. Mitochondrial DNA analysis for the pathogenic mutations at positions 3243, 3271, and 8344 was negative. Computed tomography showed generalised atrophy but no intraparenchymal abnormality. Carotid angiography was normal. Nerve conduction studies showed sensory action potentials of reduced or undetectable amplitude, and somatosensory evoked potentials were absent at the cortex but normal at the brachial plexus. Serial EEGs showed continuous polyspike and slow activity $(1-3 \mathrm{~Hz})$ over the right temporo-occipital and posterior parietal regions. Two weeks after admission her liver function tests became abnormal and sodium valproate treatment was stopped. She developed progressive liver failure, however, with jaundice and coagulopathy. Her seizures were difficult to control and she became less responsive. She died one month later, eight months after her first symptoms.

\section{Pathological features}

At postmortem there were numerous skin petechiae over her chest and abdomen, multiple intraparenchymatous petechiae in the lungs, an enlarged pale liver (weight $1750 \mathrm{~g}$ ), subcapsular haematoma in the spleen, and haemorrhages in the right kidney and adrenal. The brain was small (unfixed weight $1070 \mathrm{~g}$ ), and the only abnormality detectable on sectioning was a small zone within the right 


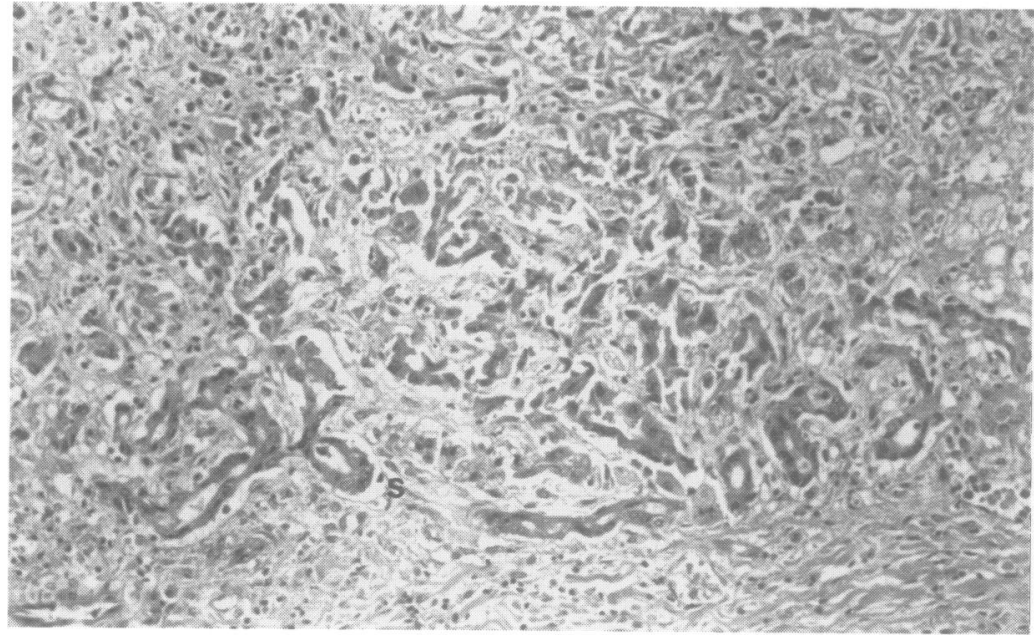

Figure 6 Postmortem liver histology: case 1, irregular bile duct like structures are embedded in fibrous tissue. The few residual hepatocytes show steatosis $(S)$.

Haematoxylin-eosin originally $\times 180$.

calcarine cortex, which was thinned, granular, and discoloured.

Microscopic examination showed widespread although mild cortical abnormality in both hemispheres. Most areas including the left striate cortex showed only moderate astrocytosis superficially with some neuronal loss. There were many Alzheimer type II astrocytes. By contrast, the right striate and peristriate cortex and a small zone in the right posterior parietal lobe were severely damaged, depleted of neurons in all but the deepest laminae, and replaced by plump astrocytes and fibrillary gliosis. Gliosis extended into the $\mathrm{U}$ fibres beneath the striate cortex. Both Ammon's horns showed moderate neuronal loss and gliosis from dentate fascia, endplate, and Sommer sector. Both thalami showed striking microvacuolation, neuronal loss, and gliosis, as did the cerebellar dentate nucleus associated with myelin depletion from its hilum. Other findings were neuronal loss with gliosis in both lateral geniculate nuclei, in the inferior olives, and in the cerebellar cortex with preservation of basket axonal plexuses and spongiosis in juxtacortical cerebellar white matter, degeneration of the gracile tract throughout its length, and numerous nodules of Nageotte in dorsal root ganglia. The liver showed cholestasis, chronic inflammation, and disorganisation of normal architecture with numerous duct like structures and surviving hepatocytes displaying microvesicular fatty change embedded in fibrous tissue.

\section{Discussion}

These patients presented with visual or sensory symptoms and signs, focal, multifocal, and generalised seizures that were difficult to control, and, eventually, hepatic failure. Death occurred within eight months of onset. Both gave rise to substantial diagnostic problems. Case 1 was initially thought to have a vascular disorder, but the subsequent progression made this unlikely. Viral encephalitis was considered in each patient, although there was no supporting evidence from CSF analyses and viral antibody titres. A metabolic disorder seemed more likely with the inexorable deterioration that occurred. A possible diagnosis of mitochondrial encephalopathy was pursued in patient 2. Although this could not be entirely excluded, there was no evidence of mitochondrial disease on muscle biopsy, relevant mitochondrial DNA mutations were not detected, and CSF lactate concentrations were normal. The correct diagnosis was made only at necropsy. From a morphological standpoint the neuropathological and hepatic changes in our patients are closely similar to those in infants and children with PNDC. Cerebral cortical destruction is often patchy, and characteristically most severe in striate cortex, differing from hypoxic, postepileptic, or hepatotoxic lesions in its lack of laminar accentuation and tendency to spread continuously through the depth of the cortex. ${ }^{14}$ Furthermore, the hepatic findings are typical for PNDC with pronounced bile duct proliferation, fatty change, and fibrosis. Pancreatitis (which occurred in patient 1) has also been described previously in PNDC. ${ }^{1}$

In most children with PNDC, psychomotor retardation or failure to thrive and hypotonia in infancy are followed by intractable seizures and liver failure. Liver function tests may be abnormal even at the time of the first fit and therefore antedate anticonvulsant treatment. ${ }^{5}$ Despite this, many children with PNDC are thought to have drug hepatotoxicity, notably to sodium valproate, as was the case in our patients; several published reports include such cases. ${ }^{89}$ The addition of thiopentone sodium coincided strikingly with the onset of hepatic failure in patient 1 . It is possible that the metabolic defect underlying PNDC predisposes to drug related hepatotoxity, but there are several notable examples of PNDC with liver disease with identical necropsy findings in pairs of siblings where only one was treated with valproate. ${ }^{110}$

The disease typically presents in infancy, rarely after five years, and is rapidly progressive with death mostly before the age of $3 .^{1}$ The presentation of our two cases at 17 and 18 years of age is therefore remarkable, but not unique. In one German family three children succumbed to PNDC with liver disease. ${ }^{11} 12$ The younger girls presented at 6 and 13 years with sudden onset of seizures and visual hallucinations, and their elder brother exhibited behavioural changes in his late teens and when 25 years old after a week's prodrome of fever and headache, had flickering and double vision, increasing confusion, and status epilepticus. He died after a four year illness characterised by tonic, clonic, and myoclonic epilepsy, striking visual hallucinations, hemianopia, gaze paresis, hemiparesis, and eventually tetraparesis. ${ }^{12}$

Unlike young children with PNDC, in whom there is usually an indeterminate period of psychomotor retardation or failure to thrive and hypotonia before seizures appear, our patients had no pre-existing neurological or developmental disorder. About $25 \%$ of the children have presented in rather 
similar fashion to our patients with explosive onset of epilepsy as the first symptom,' sometimes about a week after vaccination or nonspecific viral infection. In retrospect, the clinical, EEG, and imaging features of our patients were all compatible with a diagnosis of PNDC. The neurological disorder was similar to that seen in children, including clinical evidence of cortical visual dysfunction. ${ }^{15}$ One unusual clinical feature was the presence of a mild axonal sensory neuropathy, not previously reported in PNDC; however sensory tract degeneration in the cord has occasionally been found at necropsy. ${ }^{1}$

Characteristic electrophysiological features have been described in children with PNDC, with striking and unusual high amplitude slow activity and polyspikes in the $\mathrm{EEG}^{6}$; both our cases were remarkably similar. This EEG picture usually emerges when focal seizures become prominent, but it is notable that the abnormalities persisted in patient 1 when she developed generalised convulsive status. The posterior predominance of the abnormality in EEG and the asymmetry of the visual evoked potentials reflect the predilection of the pathology for the occipital cortex. The lesions seen in the thalamus and striate cortex on MRI correspond with the pathological abnormalities found in these regions. Radiological appearances in both our cases were similar, but are not specific, for they can accompany infarction or mitochondrial encephalomyopathy. In paediatric patients with PNDC transient changes in the occipital white matter are common, ${ }^{7}$ and these were also found here. The transient low density changes seen on CT in children, however, could not be correlated with the relatively slight abnormalities usually seen postmortem and thought to be secondary to cortical degeneration. ${ }^{7}$ The finding of a large but well circumscribed area of white matter destruction in the occipital lobe in patient 1 is therefore of interest in relation to the imaging abnormalities documented in life.

The main purpose of this report is to draw attention to the possibility of PNDC causing a subacute encephalopathy with prominent seizures in young adults. The characteristic clinical picture reported here should suggest the diagnosis, especially if there is impaired hepatic function. Data from EEG and imaging, and perhaps liver biopsy, are also helpful in making this diagnosis premortem, with practical and prognostic implications for management, and genetic considerations are also important as there is good evidence that PNDC is an autosomal recessive disorder, ${ }^{1}$ with a $25 \%$ recurrence risk to siblings.

We thank Dr GD Schott for his advice and for allowing us access to the notes of his patient (case 1) and Dr BE Kendall for helpful discussions of the neuroimaging.

1 Harding BN. Progressive neuronal degeneration of childhood with liver disease (Alpers-Huttenlocher syn-
drome): a personal review. $f$ Child Neurol 1990;5 drome):

2 Wefring KW, Lamvik JO. Familial progressive poliodystrophy with cirrhosis of the liver. Acta Paediatrica Scand 1967;56:295-300.

3 Huttenlocher PR, Solitare GB, Adams G. Infantile diffuse cerebral degeneration with hepatic cirrhosis. Arch Neurol 1976;33:186-92.

4 Harding BN, Egger J, Portmann B, Erdohazi $M$. Progressive neuronal degeneration of childhood with liver disease. Brain 1986;109:181-206.

5 Egger J, Harding B, Boyd SG. Progressive neuronal degeneration of childhood (PNDC) with liver disease. Clin Pediatr (Phila) 1987;26:167-73.

6 Boyd SG, Harden A, Egger J, Pampiglione G. Progressive neuronal degeneration of childhood with liver disease ("Alpers' disease"): characteristic neurophysiological ("Alpers' disease"): characteristic neu
features. Neuropediatrics 1986;17:76-80.

7 Kendall BE, Boyd SG, Egger J, Harding BN. Progressive neuronal degeneration of childhood with liver disease. Neuroradiology 1987;27:174-80.

8 Rolles CJ. Hepatic injury with sodium valproate: a reappraisal with special reference to children. $\mathrm{Br} f$ Clin Pract 1983;27(suppl):72-8.

9 Sheffner D, König S, Rauterberg-Roland I, Kochen W, Hoffmann WJ, Unkelbach ST. Fatal liver failure in 16 children with valproate therapy. Epilepsia 1988;29. 530-42.

10 Lenn NJ, Ellis WG, Washburn ER, Ruebner B. Fatal hepatocerebral syndrome in siblings discordant for exposure to valproate. Epilepsia 1990;31:578-83.

11 Klein H, Dichgans J. Familiäre juvenile glio-neuronale Dystrophie: akut beginnende progressive EncephaloDystrophie: akut beginnende progressive Encephalo-
pathie mit rechtsseitigen occipito-parietalen Herdpathie mit rechtsseitigen occipito-parietalen Herd-
symptomen und Status Epilepticus. Archiv für Psychiatrie symptomen und Status Epilepticus. Archiv
und Nervenkrankheiten 1969;712:400-22.

12 Bohnert B, Noetzel H. Beitrag zur familiären spongiösen glioneuralen Dystrophie. Archiv für Psychiatrie und Nervenkrankheiten 1974;218:353-68. 